\title{
Apoyo psicológico en oncología radioterápica
}

\section{J. A. Cruzado}

\section{Introducción}

El tratamiento del cáncer mediante radioterapia supone para el paciente y sus familiares una oportunidad de control, mejoría o curación de esta enfermedad; sin embargo, al mismo tiempo implica afrontar importantes retos que pueden afectar a su calidad de vida y estado emocional. Estos desafíos pueden agruparse principalmente en las siguientes áreas:

- Información, comprensión e incertidumbre acerca de la radioterapia.

- Problemas de comunicación con el personal sanitario.

- El miedo de los aparatos y administración del tratamiento.

- Reacciones emocionales desadaptativas:

- Ansiedad.

- Depresión.

- Hostilidad.

- Sentimientos de culpa:

- Aislamiento.

- Negación, etc.

- Efectos de la radioterapia o la patología del cáncer:

- Fatiga.

- Náuseas y vómitos.

- Dolor.

- Irritación de la piel.

- Problemas sexuales.

- Infertilidad.

- Anorexia.

- Problemas de la vida diaria y administración de tiempo, que surgen debido a la patología o al tratamiento.

- Comunicación y apoyo adecuado de la pareja, hijos y padres al paciente.

Con el objeto de prevenir problemas de desadaptación y déficit en calidad de vida es necesario llevar a cabo un adecuado protocolo de evaluación psicológica. La experiencia llevada a cabo en los Servicios de Radioterapia Oncológica

Master en Psicooncología

Universidad Complutense de Madrid del Hospital Ramón y Cajal, Puerta de Hierro y Central de la Defensa aconsejan llevar a cabo el siguiente protocolo de evaluación:

\section{Entrevista clínica:}

- Información y comprensión de la enfermedad.

- Estado emocional y preocupacione.

- Interacción con el equipo.

- Afectación familiar.

- Antecedentes psicopatológicos.

- Estrés previo.

- Afrontamiento.

- Competencias y recursos propios.

\section{Evaluación de la ansiedad y depresión}

- Escala de ansiedad y depresión hospitalaria.

- Escala de Ansiedad Estado-Rasgo (STAI).

- Inventario de Depresión de Beck.

\section{Calidad de vida: EORTC o FACT}

Las estrategias de intervención psicológica en Radioterapia Oncológica fundamentalmente son las siguientes:

\section{Psicoeducación}

- Información y asesoramiento acerca del procedimiento de radioterapia.

- Miedos y temores usuales en radioterapia.

- Esta información es verbal y escrita (folletos informativos) e incluye exposición previa a los estímulos temidos.

\section{Consejo psicológico y orientación}

Sobre estrategias para superar problemas de información, dudas y necesidades con el equipo médico mediante una comunicación asertiva.

\section{Control del miedo y la ansiedad ante la radioterapia}

- Relajación.

- Hipnosis.

- Control de atención.

- Inoculación de estrés. 


\section{Afrontar emociones negativas}

- Identificar las emociones negativas y sus síntomas.

- Expresar las emociones.

- Identificar situaciones y pensamientos provocadores.

\section{Planificación de activides de placer y dominio}

- Modificación del diálogo interno.

- Reestructuración cognitiva.

\section{Técnicas específicas de control de efectos colaterales}

- Información.

- Solución de problemas.

- Inoculación de estrés.

\section{Habilidades de comunicación y de interacción social}

\section{Investigación empírica}

Con el objeto de planificar adecuadamente y mejorar la atención psicológica es necesario llevar a cabo una investigación sobre la calidad de vida a lo largo del tratamiento mediante radioterapia. En este sentido se llevó a cabo un estudio desde noviembre de 2002 hasta junio de 2003 en el Servicio de Radioterapia del Hospital Ramón y Cajal.

\section{Objetivos}

- Determinar cuáles son los niveles de ansiedad y depresión lo largo del proceso de radioterapia externa.

- Determinar los niveles de calidad de vida a lo largo del proceso de radioterapia oncológica medidos por la Escala EORTC.

\section{Muestra}

La muestra está constituida por 55 personas: 35 mujeres y 20 varones, edad media 44 años (intervalo 30-72 años), con diversos cánceres: mama, ginecológicos, próstata, colon, gástrico, pulmón, óseo y SNC.

\section{Variables e instrumentos}

- Ansiedad y depresión medidas por la Escala de Ansiedad y Depresión Hospitalaria (HAD).

- Calidad de vida medida por el cuestionario de la EORTC: EORT QLQ-C30, Escala de 30 ítems que mide las siguientes dimensiones:

- Calidad de vida global.

- Funcionamiento físico.

- Funcionamiento de rol.

- Funcionamiento emocional.

- Funcionamiento cognitivo.

- Funcionamiento social.

- Escalas de síntomas: fatiga, náuseas y vómitos, dolor, diseña, insomnio, pérdida de apetito, estreñimiento, diarrea y dificultades financieras.

\section{Procedimiento}

Se toman medidas de las variables dependientes antes de la administración de la sesión de radioterapia, una vez por semana, previamente al comienzo de la aplicación del tratamiento.

\section{Resultados}

En relación a la ansiedad se observa que al comienzo es muy elevada y va descendiendo a medida que se prolonga el tratamiento (Fig. 1). Es posible que los pacientes se vayan habituando a la aplicación del procedimiento con el paso del tiempo.

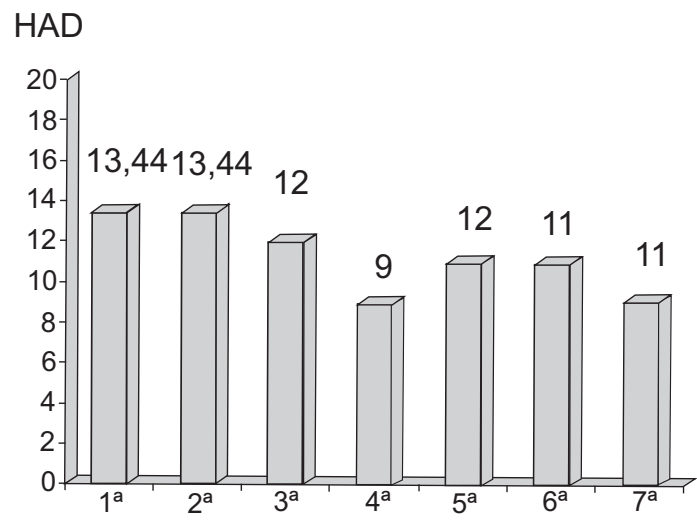

Semanas de tratamiento

Fig. 1. Ansiedad.

Con respecto a la medida de depresión se observa una elevada puntuación al principio, que pone de relieve que el paciente inicia el tratamiento con sentimientos de indefensión y tristeza; después disminuyen, si bien en las últimas sesiones se produce una cierta elevación en la media con claro significado clínico de que están presente sentimientos de tristeza en los pacientes (Fig. 2).

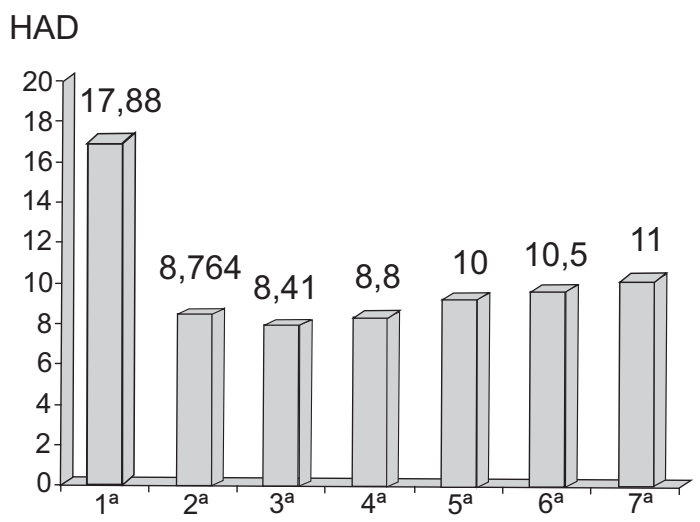

Semanas de tratamiento

Fig. 2. Depresión. 
En cuanto a la medida de calidad de vida global medida por el cuestionario de la ORTC, se observan niveles bajos al principio y después se asiste a una elevación en las puntuaciones, aunque siempre en niveles medios (Fig. 3).

\section{QLC-C30}

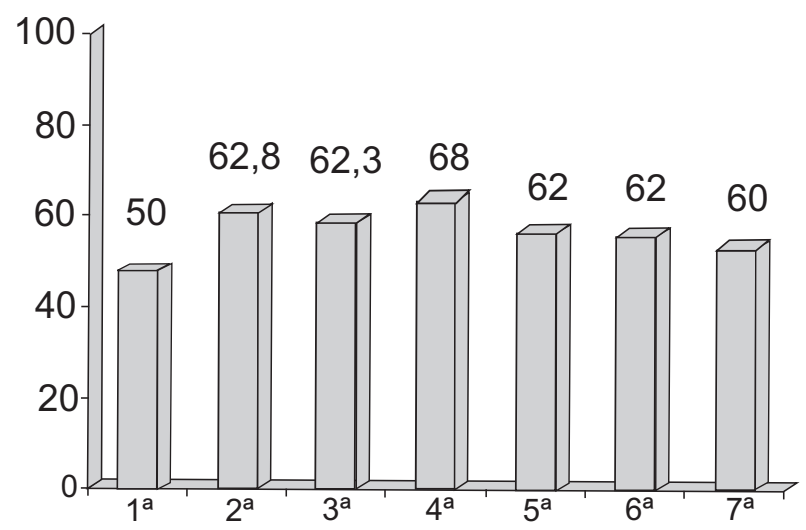

Semanas de tratamiento

Fig. 3. Calidad de vida global.

En relación al funcionamiento físico (Fig. 4), funcionamiento de rol (ver Figura 5) funcionamiento emocional (Fig. 6), cognitivo (Fig. 7) y social (Fig. 8) se mantienen en niveles medio-alto, excepto en las dos últimas semanas en que se puntúa más bajo

\section{QLC-C30}

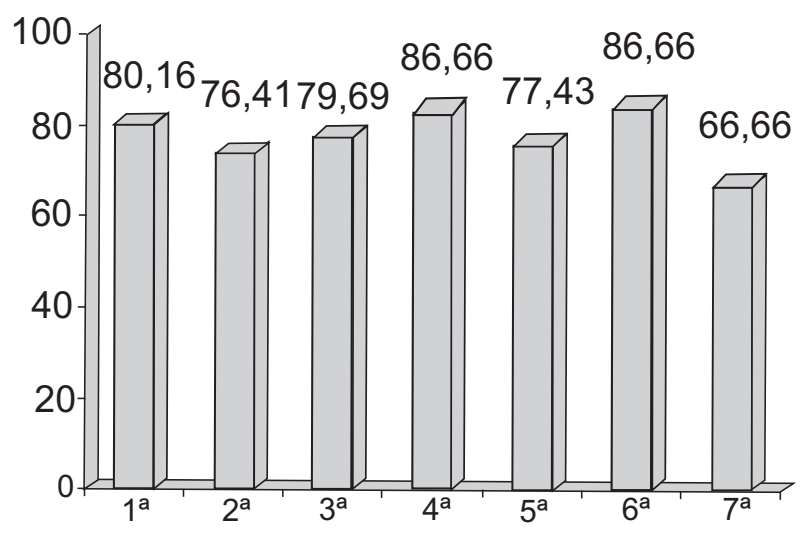

Semanas de tratamiento

Fig. 4. Calidad de vida. Funcionamiento físico.

En cuanto a los síntomas (Fig. 9), se observa un aumento de sintomatología relacionada con la fatiga sobre todo en las últimas semanas del tratamiento.
QLC-C30

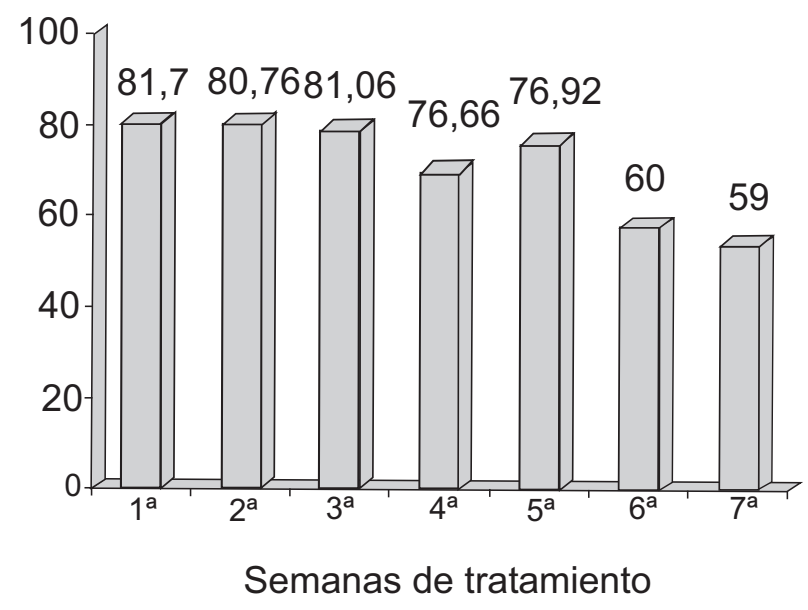

Fig. 5. Calidad de vida. Funcionamiento rol.

\section{QLC-C30}

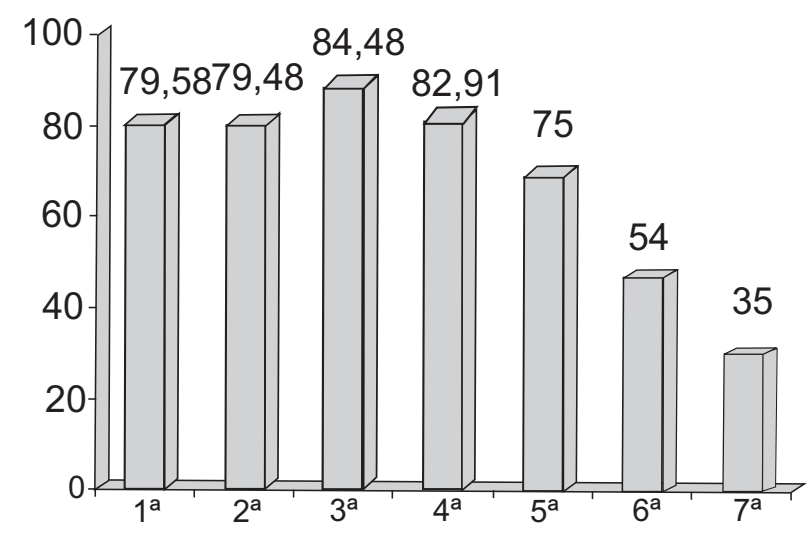

Fig. 6. Calidad de vida. Funcionamiento emocional.

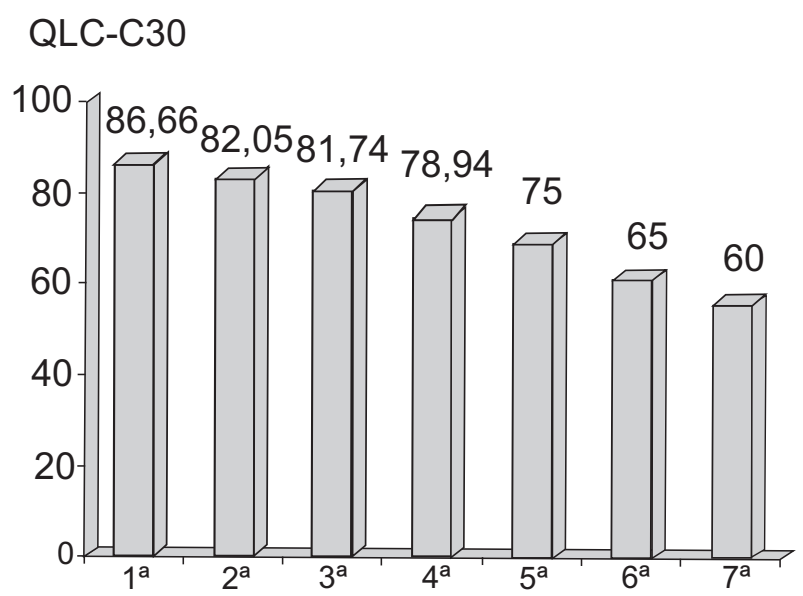

Semanas de tratamiento

Fig. 7. Calidad de vida. Funcionamiento cognitivo. 


\section{QLC-C30}

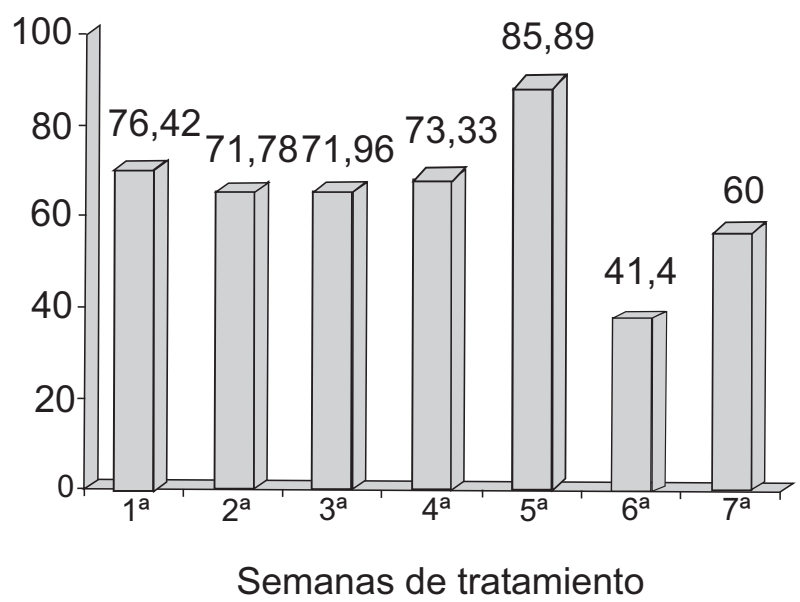

Fig. 8. Calidad de vida. Funcionamiento social.

\section{Conclusión}

La calidad de vida y el estado emocional son variables que han de ser evaluadas a lo largo de todo el proceso de aplicación de la radioterapia. Los datos muestran cómo previamente al comienzo del tratamiento la ansiedad y depresión son muy elevadas, después disminuyen ambas a medida que el paciente se habitúa a los tratamientos.

\section{QLC-C30}

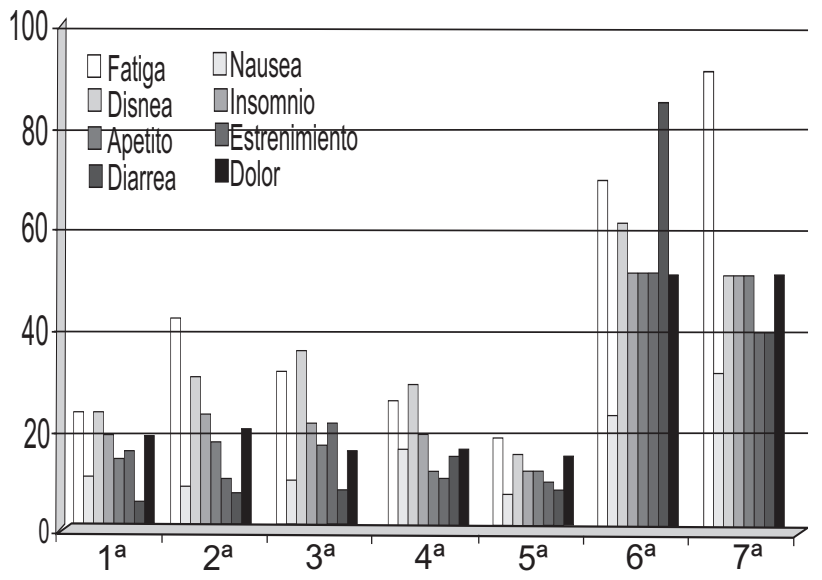

Fig. 9. Calidad de vida. Síntomas.

La calidad de vida se mantiene en niveles medios, al principio y en las sesiones intermedias; en las dos últimas semanas se produce una disminución en los niveles globales de calidad de vida y en las diferentes áreas de funcionamiento, pero sobre todo hay un aumento en los síntomas, tales como la fatiga y por ello las puntaciones en depresión vuelven a aumentar significativamente. 